\title{
Preventive treatment for migraine in primary care, a population-based study in the Netherlands
}

0(0) $1-9$

(C) International Headache Society 2013

Reprints and permissions:

sagepub.co.uk/journalsPermissions.nav DOI: $10.1177 / 0333102413490343$ cep.sagepub.com

$@$ SAGE

\section{F Dekker', JP Dieleman ${ }^{2}$, A Knuistingh Neven', MD Ferrari ${ }^{3}$ and WJJ Assendelft'}

\begin{abstract}
Background: Preventive treatment of migraine contributes to reducing the impact of migraine but its extent of use in routine care is unknown.

Objective: The objective of this article is to assess current use, previous use, duration and course of preventive treatment of migraine in Dutch general practice.

Methods: We conducted a retrospective cohort study, for the period between 1997 and 2007, in the Interdisciplinary Processing of Clinical Information (IPCI) database, a GP research database in the Netherlands (source population of more than half a million subjects). All prevalent and incident migraine patients $(N=7367)$ were included.

Results: About 13\% of all migraine patients currently use preventive therapy and almost half of migraine patients have prior use. Of those starting with preventive treatment, $56 \%(95 \% \mathrm{Cl}: 54.3-64.7)$ still used it after nine months. There was a long delay between migraine diagnosis and preventive treatment start. Forty-four percent $(95 \% \mathrm{Cl} 43.0-45.7)$ started preventive therapy in the study period.

Conclusion: This large primary-care database study shows that a limited number of patients are current users of preventive treatment, but many patients have prior use. After diagnosis there is often an extended time before preventive treatment is applied. Also there is often only one attempt. The continuation in time seems appropriate. Preventive therapy in migraine still deserves focus.
\end{abstract}

\section{Keywords}

Migraine, headache, prophylaxis, preventive treatment, incidence, medication-overuse headache

Date received: 25 January 2013; accepted: 21 April 2013

\section{Introduction}

Migraine is a common, chronic, incapacitating neurovascular disorder characterized by attacks of severe headache, autonomic nervous system dysfunction, and in some patients, an aura involving neurological symptoms (1). The mean one-year prevalence of migraine in adults aged $18-65$ years is more than $10 \%(10.9 \%$ in the Americas and $14.8 \%$ in Europe) (2).

An important treatment goal is reducing the attack frequency. Preventive treatment includes beta blockers and anti-epileptics and is recommended in guidelines (3-6). In the Netherlands preventive treatment with propranolol or metoprolol is recommended for patients with an attack rate of two or more attacks per month $(3,4)$.

Several studies into preventive treatment for migraine suggest large under-treatment. A telephone survey in the United States (US) showed that only $13 \%$ of migraine patients who qualified for preventive treatment actually received treatment $(7,8)$. An earlier questionnaire study showed similarly low numbers $(5 \%$ and $12.4 \%$ ) for preventive treatment use $(9,10)$, although an additional $17.2 \%$ of patients used medication with

\footnotetext{
'Public Health and Primary Care, Leiden University Medical Center, the Netherlands

${ }^{2}$ Department of Medical Informatics, Erasmus Medical Centre, the Netherlands

${ }^{3}$ Neurology, Leiden University Medical Center, the Netherlands

Corresponding author:

F Dekker, Public Health and Primary Care, Leiden University Medical Center, Post zone VO-P, P.O. Box 9600, 2300 RC Leiden, the

Netherlands.

Email: f.dekker@lumc.nl
} 
potential antimigraine effects for other indications (10). In France use of preventive treatment was estimated at $6 \%$ (11). In a small Dutch study in general practice (GP), $8 \%$ of migraine patients used preventive medication (12). Recently, we described a Dutch pharmacy record study comprising 6.2 million people, showing that $19 \%$ of the triptan users at some time also take preventive medication (13). Most studies on preventive migraine treatment use suffer from methodological shortcomings and differ among each other. In pharmacy records, the indication for drug prescribing is usually lacking, as a result of which the medication may be prescribed for other reasons (like hypertension). Questionnaire studies in the general population also may have included inappropriate migraine diagnoses and misclassify frequent attack treatment as preventive treatment (11).

For patients, the frequency of migraine attacks is the most important consideration in the decision for preventive treatment (12). Preventive treatment is also indicated when exuberant use of attack medication is imminent and when there is a risk of medication-overuse headache $(\mathrm{MOH})$, which has a population prevalence of $4 \%$ in adults $(14,15)$. Data on triptan use in the Netherlands suggest that triptans possibly cause more headaches than they cure (13).

For policy-making and optimizing routine care, a valid estimate of the proportion of migraine patients receiving preventive treatment is necessary. We performed a population-based cohort study in a GP research database in the Netherlands to gain more insight into preventive treatment of migraine in primary care.

\section{Methods}

\section{Setting}

The study was conducted using the Integrated Primary Care Information (IPCI) database, a longitudinal GP research database in the Netherlands. The IPCI database is maintained by the Department of Medical Informatics of the Erasmus MC, University Medical Centre Rotterdam in the Netherlands. The database contains longitudinal data from computer-based patient records of more than 150 GPs throughout the Netherlands. Presently, the database comprises data on more than 800,000 subjects, of whom the age and gender distribution is similar to the Dutch population. The registration uses the International Classification of Primary Care (ICPC) $(16,17)$ to register patient symptoms and diagnoses, although these can also be entered as free text. Prescription data include product name, quantity dispensed, dosage regimens, strength and indication. As of 1996, drugs are coded according to the Anatomical Therapeutic Chemical (ATC) classification scheme recommended by the World Health Organization (WHO) (18). GPs who participate in the IPCI project are not allowed to use paper-based records.

The IPCI database system complies with European Union guidelines on the use of medical data for medical research and has been proven valid for pharmaco-epidemiological research (19). The Scientific and Ethical Advisory Group of the IPCI project approved the study (number 05/92).

\section{Study period and study population}

We used data of all individuals with at least one year of follow-up data available in the database between January 1996 and December $2007(n=478,584)$. One year of data was needed to validly assess medical history and treatment history. Study entry was defined as the date at which one year of follow-up was accumulated or January 1, 1996, whichever was latest. Followup lasted until the end of the study period, end of patient registration with GP practice, death or last IPCI data delivery, whichever came first. Within the study population we identified a sub-cohort of patients with newly detected migraine (incident diagnosis).

\section{Ascertainment of migraine}

GPs have a diagnostic code for migraine (ICPC-code N89). Additional potential cases were identified in the database through an inclusive string search on free text ('migrain*'). The presence and date of diagnosis of migraine were evaluated by a manual review of the electronic patient record (EPR) of all the potential cases by the principal investigator (FD). Our case definition relied on the International Headache Society (IHS)-classification (20). As typical migraine symptoms we considered attack frequency, duration of the headache, (unilateral) location, pulsating quality, moderate or severe pain intensity, aggravation by or causing avoidance of routine physical activity (e.g. walking or climbing stairs), nausea or vomiting, photophobia and phonophobia, and the existence of precipitating aura, shooting, or burning pain. We accepted GP diagnoses if they recurred in the patient record and if typical migraine symptoms were present.

The index date was defined as the date of diagnosis of migraine. If the index date was on or after the start of follow-up, the migraine was classified as incident. In other cases the migraine was considered as prevalent.

We distinguished two categories of migraine as abstracted from the database, namely "uncertain migraine" and "certain migraine." Uncertain migraine comprised patients who expressed symptoms of 
migraine or had sporadic attacks, or were labeled by the physician as having migraine, but visited the GP only once for that reason. These patients had incomplete symptoms or were only once indistinctly associated with migraine in the whole research period (no preventive therapy). Uncertain migraine was not included in our analysis. This category has to be distinguished from 'probable migraine' from the IHS classification (20). Migraine was classified as "certain migraine" if the headache features corresponded to the IHS criteria for migraine (20). We further detailed certain migraine according to presence of aura and considered menstrually related migraine (MRM) as a subgroup of certain migraine. Patients with typical aura without headache $(\mathrm{AWoH})$ and patients with $\mathrm{MOH}$ were considered separate from certain migraine.

\section{Migraine treatment}

Treatment details extracted from the database comprised the date of prescribing, full ATC code, drug name, strength, dose instructions and primary indication for the prescription. As attack treatment we considered drugs mentioned in Dutch guidelines (paracetamol, acetylsalicylic acid, nonsteroidal antiinflammatory drugs (NSAIDs), triptans, ergots) and as preventive treatment we considered agents that are mentioned in Dutch guidelines or are officially approved preventive treatment (beta blockers without intrinsic sympathomimetic activity, valproic acid, topiramate, pizotifen, amitriptyline and flunarizine) $(3,4)$.

\section{Analysis}

We calculated age- and gender-specific incidence rates of different types of migraine by dividing the number of incident cases by the total accumulated follow-up time. Ninety-five percent confidence intervals $(95 \%$ CI) were calculated assuming a Poisson distribution. We used Kaplan-Meier analysis to calculate the time to preventive treatment start following migraine diagnosis in patients who had not used any of the preventive treatment agents before. Kaplan-Meier analysis was also used to estimate the 11-year cumulative incidence of treatment initiation following migraine diagnosis.

To assess duration of treatment we assessed the proportion of patients still using the initial medication in periods of three, six, nine and 12 months following treatment start. Proportions were calculated by dividing the number of patients remaining on initial treatment by the number of patients still in follow-up at the end of the concerned period. All the analyses were conducted using SPSS for Windows version 15.0 (SPSS Inc, Chicago, IL, USA).

\section{Results}

The total amount of follow-up time for the 472,033 subjects in the study population without migraine at baseline was $1,855,904$ person years, with an average of 3.9 person years per subject (standard deviation (SD): 2.99). We identified 7525 first-time diagnoses of migraine or related headache disorders, of which 5134 were certain migraine, 2081 uncertain migraine, 152 $\mathrm{AWoH}$ and $158 \mathrm{MOH}$ (Figure 1). MRM was found in 712 patients (Table 1). Migraine patients were predominantly female $(75 \%)$ and mostly between 30 and 40 years of age (Table 1). The overall incidence rate of migraine over the entire study period, including uncertain cases, was 4.05 per 1000 person years $(95 \% \mathrm{CI}$ : 3.96-4.15). Considering certain migraine only, the incidence rate was 2.8 per 1000 person years $(95 \%$ CI: 2.7-2.8).

\section{Treatment of migraine}

Out of 5134 migraine patients, $684(13.3 \%, 95 \%$ CI: 12.4-14.3) received a preventive treatment prescription at any time during the study period, most of them without ever receiving attack treatment in the study period (no attack treatment out of 684: $n=501,73.2 \%, 95 \%$ CI: 69.7-76.5) and consisting of a single type $(n=552$, 80.7\%, 95\% CI: 77.5-83.6). Of all patients receiving preventive therapy, 21 patients $(3.1 \%, 95 \%$ CI: 2.0 4.7) had two or more preventive therapies (Figure 1).

Among patients diagnosed as having migraine with aura, $11.2 \%$ (95\% CI: 9.40-13.30) were prescribed preventive treatment. Migraine patients without aura receiving preventive treatment comprised $13.2 \%(95 \%$ CI: 8.3-20.1), and in patients for whom the presence of aura was not mentioned, the percentage of those receiving preventive treatment was $13.1 \%$ (95\% CI: $12.1-$ 14.2). The odds ratio (OR) for having received preventive treatment in patients with aura versus patients not having aura or aura not specified was 0.87 (95\% CI: 0.70-1.08)). AWoH was recorded for 152 cases, of whom $24(15.8 \%, 95 \%$ CI: 10.6-222.8) received preventive treatment (Table 2).

Of the 1936 migraine patients using triptan, 92 (4.8\%, CI: 3.87-5.52) also used preventive medication. Of the 1080 NSAID users, $45(4.2 \%, 95 \%$ CI: 3.09 5.58) also had preventive medication.

The average age for those receiving prescribed attack treatment was 38.1 (SD 14.6) years and for preventive treatment 42.3 (SD 15.4) years.

The Kaplan-Meier analysis showed that first-time preventive treatment prescriptions were issued mostly within the first two years following diagnosis but continued to be issued at a lower steady rate thereafter (Figures 2 and 3). The cumulative incidence of ever 


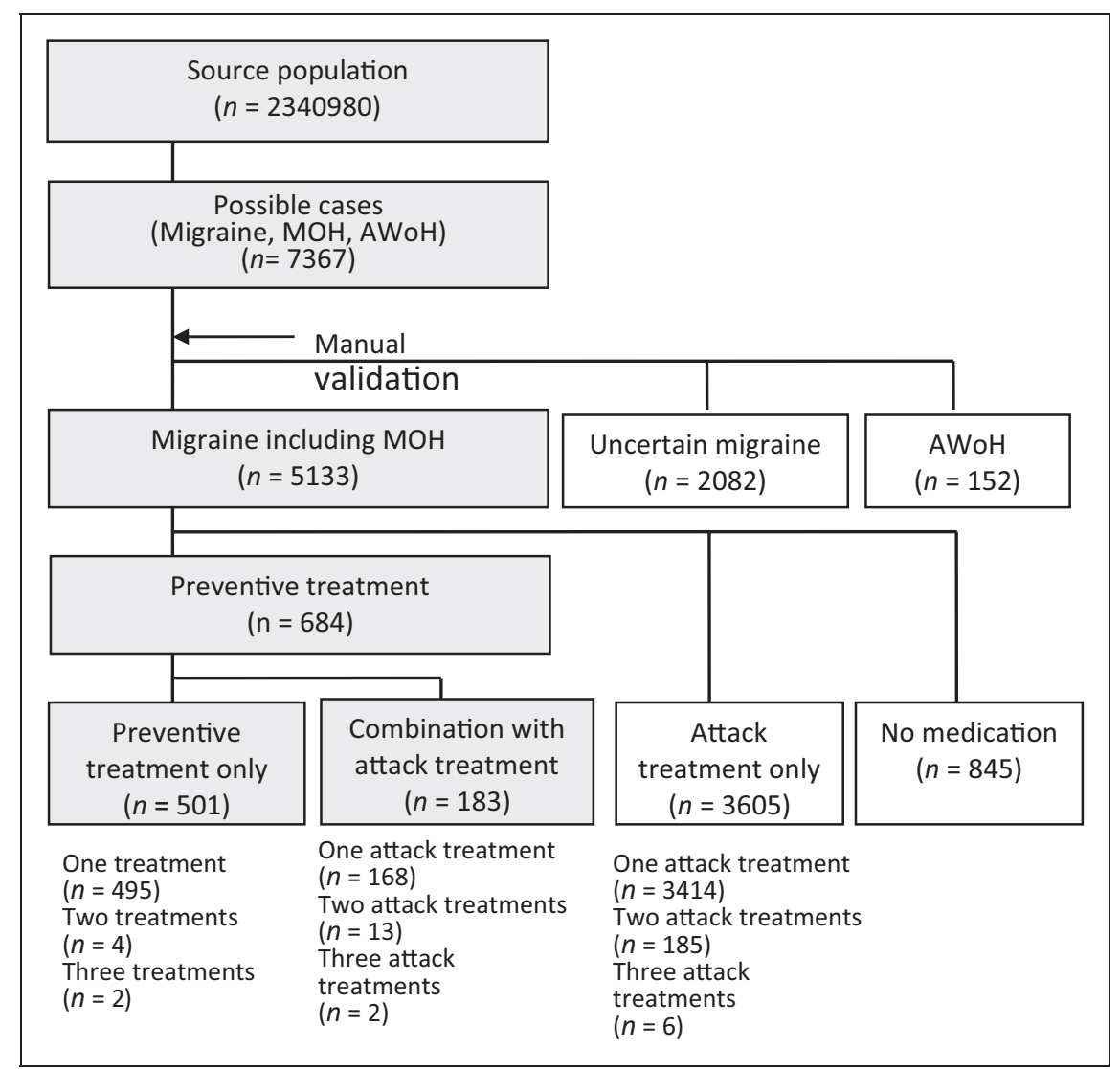

Figure I. Process of identification of preventive migraine cases in the Integrated Primary Care Information (IPCI) database. n: number; $\mathrm{MOH}$ : medication-overuse headache; $\mathrm{AWoH}$ : aura without headache.

Table I. Characteristics of incident migraine, medication-overuse and typical aura without headache in general practice.

\begin{tabular}{|c|c|c|c|c|c|c|c|}
\hline & Total & $\begin{array}{l}\text { Total cases migraine } \\
\text { (certain }+ \text { uncertain) }\end{array}$ & $\begin{array}{l}\text { Uncertain } \\
\text { migraine }\end{array}$ & $\begin{array}{l}\text { All certain } \\
\text { migraine }\end{array}$ & $\begin{array}{l}\text { Subgroup } \\
\text { MRM }^{\mathrm{a}}\end{array}$ & $\mathrm{AWoH}^{\mathrm{b}}$ & $\mathrm{MOH}^{\mathrm{c}}$ \\
\hline Total & $N=2,340,980 n(\%)$ & $N=7367 n(\%)$ & $N=208 \mid n(\%)$ & $N=4975 n(\%)$ & $N=712 n(\%)$ & $N=152 n(\%)$ & $N=158 n(\%)$ \\
\hline \multicolumn{8}{|l|}{ Sex } \\
\hline Male & I,I62,830 (49.7) & $1859(25.2)$ & $566(26.7)$ & $1213(24.4)$ & - & $55(36.2)$ & $25(15.8)$ \\
\hline Female & I,I 78,I $50(50.3)$ & $5508(74.8)$ & $1515(72.3)$ & $3762(75.5)$ & 712 & $97(63.8)$ & I 33 (84.2). \\
\hline \multicolumn{8}{|l|}{ Age } \\
\hline $0-10$ & $247,506(10.6)$ & $258(3.5)$ & $90(4.3)$ & $167(3.4)$ & - & I (0.7) & - \\
\hline $10-19$ & 274,523 (II.7) & 880 (II.9) & $256(12.3)$ & $607(12.2)$ & $36(5.1)$ & $5(3.3)$ & $12(7.6)$ \\
\hline $21-29$ & $321,827(13.7)$ & I388 (18.8) & $396(19.0)$ & $947(19.0)$ & $135(19.0)$ & $12(7.9)$ & $33(20.9)$ \\
\hline $31-39$ & $391,838(16.7)$ & I826 (24.8) & 497 (23.9) & $1238(24.9)$ & $288(40.4)$ & $34(22.4)$ & $57(36.1)$ \\
\hline $4 I-49$ & $361,684(15.5)$ & $1665(22.6)$ & $450(21.6)$ & I I 62 (23.4) & $220(30.9)$ & $30(19.7)$ & $23(14.6)$ \\
\hline $5 I-59$ & $303,424(13.0)$ & $872(11.8)$ & $249(12.0)$ & $557(\mid 1.2)$ & $33(4.6)$ & $39(25.7)$ & $27(17.1)$ \\
\hline $61-70$ & 270,737 (II.6) & $294(4.0)$ & $83(4.0)$ & $191(3.8)$ & - & $16(10.5)$ & $4(2.5)$ \\
\hline $71+$ & $|69,44|(7.2)$ & $184(2.5)$ & $61(2.9)$ & $106(2.1)$ & - & $15(9.9)$ & $2(1.3)$ \\
\hline
\end{tabular}

${ }^{a} \mathrm{MRM}$ : Menstrually related migraine is a subgroup of certain migraine. ${ }^{\mathrm{b}} \mathrm{AWoH}$ : typical aura without headache. ${ }^{\mathrm{c}} \mathrm{MOH}$ (medication-overuse headache) is not part of the total migraine (=all certain migraine). 
having used preventive treatment within a study period of 11 years (average 3.9 person years (SD: 2.99)) was 44.3\% (95\% CI: 43.0-45.7). For women this was $47.0 \%$ (95\% CI 43.4-48.6) and for male 36.1\% (95\% CI: 33.4-38.9).

The average time lapse between starting attack treatment and starting preventive treatment was 4.3 years during this 11-year study period. Patients started preventive migraine treatment at a high average age: 38.1 for males and 42.3 years for females.

Among patients starting preventive treatment, many had discontinued that treatment within three months, after which the percentage of users stabilized. The average proportion of patients still using preventive treatment after nine months of first start was 55.6\% (95\% CI: 54.9-60.1) but varied widely by treatment. Patients using beta blockers were most likely to continue treatment $(59.6 \%$ after nine months, 95\% CI: 54.3-64.7) whereas patients using pizotifen were least likely to continue $(37.0 \%$ after nine months, 95\% CI: 26.2-49.1). Among patients using beta blockers, prolonged use after nine months was $77.8 \%$ (95\% CI: $62.5-88.3$ ) for atenolol, $62.6 \%(95 \% \mathrm{CI}$ : $53.4-71.0)$ for metoprolol and $50.3 \%$ (95\% CI: 42.6-58.0) for propranolol. For patients using amitriptyline or valproic acid, the numbers were too small to make reliable estimates.
We also studied the extent to which patients continued to use initial, prescribed attack treatment. The drop in attack treatment use was much larger than the drop in preventive treatment. After nine months the proportion still receiving prescriptions for a triptan was $26.7 \%$ (95\% CI: 20.4-34.3) (not available over the counter (OTC) in the Netherlands) and for NSAIDs, 26.8\% (95\% CI: 24.5-29.3) (available OTC).

\section{Discussion}

In a large Dutch longitudinal GP research database we found that the percentage of all migraine patients receiving preventive treatment in the Netherlands was $13.3 \%$ (95\% CI: $12.4-14.3$ ). Approximately $56 \%$ of those starting preventive treatment continued on it for a prolonged period of time (i.e. nine months), suggesting good treatment effect and acceptable side effects. The duration of first preventive treatment use was much longer than that for first attack treatment. In the vast majority of patients only one type of preventive treatment was tried, which may be because the current guideline recommends only beta blockers as preventive treatment (3).

The large time lapse between starting attack- and preventive treatment of 4.3 years and the high average

Table 2. Use of preventive treatment in migraine.

\begin{tabular}{|c|c|c|c|c|c|c|c|}
\hline & \multicolumn{5}{|c|}{ Migraine (certain) } & \multirow[b]{3}{*}{$\mathrm{AWoH}$} & \multirow[b]{3}{*}{$\mathrm{MOH}$} \\
\hline & \multirow[b]{2}{*}{$A l^{\mathrm{a}}$} & \multicolumn{3}{|c|}{ With and without aura } & \multirow{2}{*}{$\begin{array}{l}\text { MRM } \\
\text { (subgroup) }\end{array}$} & & \\
\hline & & Unspecified & $\mathrm{MA}+$ & $\mathrm{MA}-$ & & & \\
\hline Total & 5134 & 3989 & 1053 & 144 & 712 & 152 & 158 \\
\hline preventive (\%) & $684(12.9)$ & $523(13.1)$ & $118(11.4)$ & $19(13.2)$ & $72(3.8)$ & $24(15.79)$ & $15(9.5)$ \\
\hline \multicolumn{8}{|c|}{ Specification preventive medication } \\
\hline Propranolol & $240(35.1)$ & $192(36.7)$ & $38(32.2)$ & $6(31.6)$ & $12(44.4)$ & $4(16.7)$ & $7(46.7)$ \\
\hline Metoprolol & $177(25.9)$ & $133(25.4)$ & $31(26.3)$ & $3(15.8)$ & 7 (25.9) & I (4I.7) & $2(13.3)$ \\
\hline Other beta blockers & $87(12.7)$ & $59(\mathrm{I} I .3)$ & $21(18.8)$ & I (5.3) & $4(14.8)$ & $6(25.0)$ & \\
\hline All-antagonists & $7(1.1)$ & $6(1.1)$ & I (0.8) & - & - & - & - \\
\hline Verapamil & $6(0.9)$ & $5(1.0)$ & I (0.8) & - & - & - & - \\
\hline Valproic acid & $16(2.3)$ & $8(1.5)$ & $4(3.4)$ & $3(15.8)$ & - & I (4.2) & $2(13.3)$ \\
\hline Topiramate & $2(0.3)$ & $2(0.4)$ & - & - & - & - & - \\
\hline Pizotifen & $80(11.7)$ & $63(12.0)$ & $16(13.6)$ & I (5.3) & - & - & $2(13.3)$ \\
\hline Clonidine & $29(4.4)$ & $23(4.4)$ & $2(1,7)$ & 4 (2I.I) & I (3.7) & I (4.2) & - \\
\hline Flunarizine & $9(1.3)$ & $7(1.3)$ & - & - & I (3.7)- & $2(83)$ & \\
\hline Methysergide & $3(0.4)$ & $2(0.4)$ & I (0.8) & - & - & - & - \\
\hline Amitriptyline & $36(5.3)$ & 31 (5.9) & $4(3.4)$ & I (5.3) & $2(7.4)$ & - & $2(13.3)$ \\
\hline
\end{tabular}

Numbers of patients (percentage). MA+: migraine with aura; MA-: migraine without aura; MRM: menstrually related migraine (as mentioned in electronic patient record (EPR), both regular and pure); $\mathrm{AWoH}$ : typical aura without headache (migraine sans migraine); $\mathrm{MOH}$ : medication-overuse headache. ${ }^{a}$ Without $\mathrm{AWoH}$, including $\mathrm{MOH}$. 


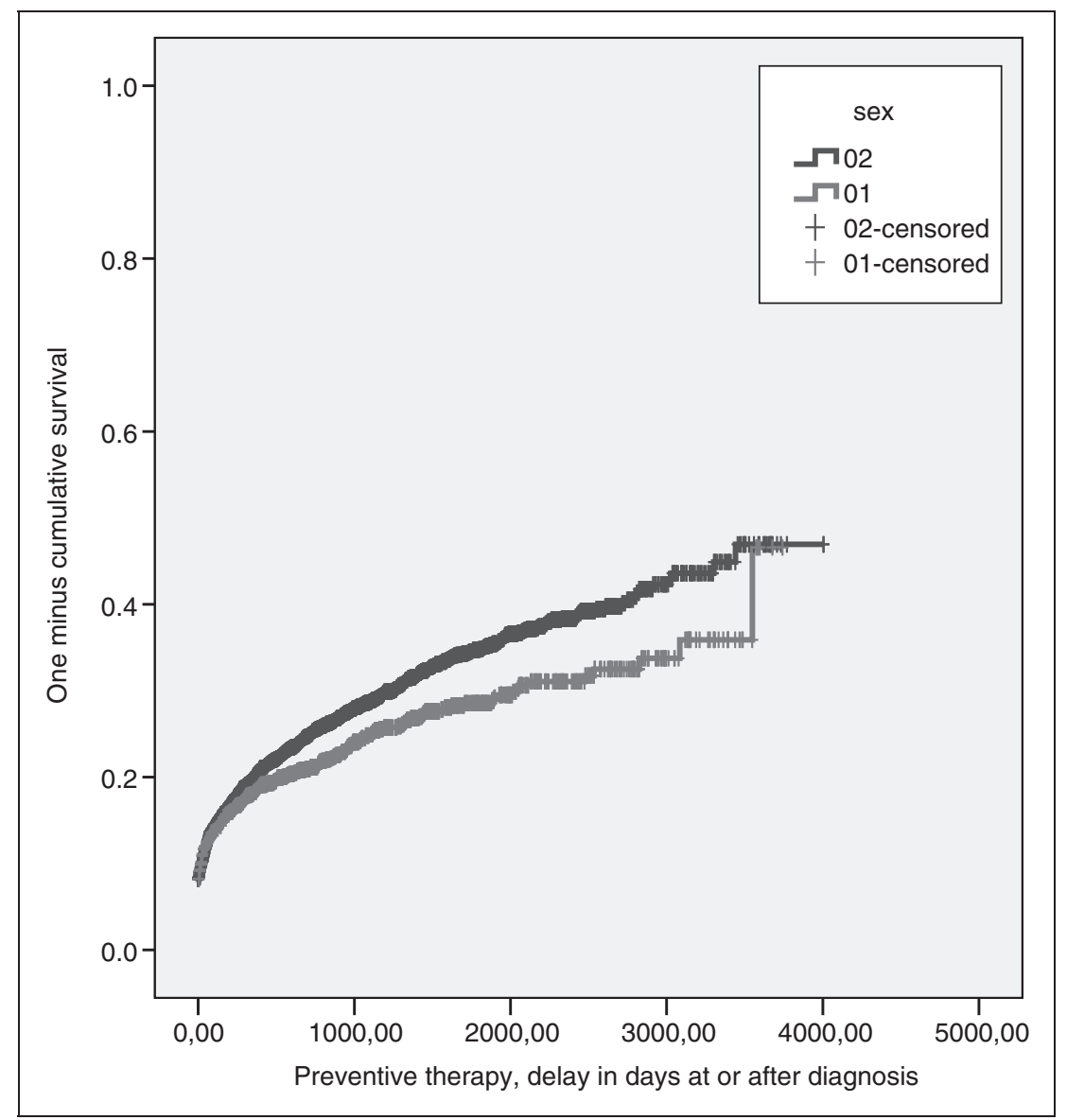

Figure 2. Kaplan-Meier graph of start of preventive treatment related to time after first diagnosis of migraine. One minus cumulative survival curve of the time span between initial migraine diagnosis and the start of preventive treatment, and the difference between males and females. 01: male, 02: female. Time is shown in days after initial diagnosis of migraine.

age at start (male 38.1 and female 42.3 years) indicates that at the start of preventive therapy the burden of migraine often is already past its highest peak throughout life (1). This underlines the reluctance to accept the daily use of preventive medication (21).

The number of new patients receiving preventive treatment remained stable over the study years. And as the incidence of new cases of migraine decreased over the years, there was a relative increase in the use of preventive treatment (data not shown).

We found no significant differences in migraine treatment prescribing between different types of migraine, or between presence and absence of aura. Neither did we find a relation between prescribing of preventive treatment and that of attack treatment (see Figure 1); only a small proportion of patients receiving preventive treatment also received prescriptions for attack treatment.

The attack frequency at which patients are recommended to start preventive therapy varies widely in guidelines $(22,23)$. The Dutch guidelines recommend preventive therapy in case of two or more attacks per month in primary care (3) or advise preventive treatment in case of three of more attacks per month in secondary care (4).

Our rate of $13 \%$ preventive treatment is in line with studies in other countries (7,9-12,24-27).

Some studies, however, reported a lower frequency of use $(25,28)$. Others have estimated that $5 \%-83 \%$ of migraine patients qualify for preventive treatment $(8,10,29)$. One study in the same population as ours claims much higher numbers for prevention (30). However, this study was performed in a high selective population of patients referred to a neurologist and as the presented data do not correspond with previous studies $(12,24,25)$, this study is of questionable importance with which to compare preventive treatment.

Our study period covers 11 years with an average of 3.9 person years' availability, whereas most previous studies reported the actual number of patients per year. It shows that when cumulated over a longer period many more patients, $44.3 \%$ of all patients, have tried preventive treatment, which is well above previous estimates $(7,9-12,24-27)$. 


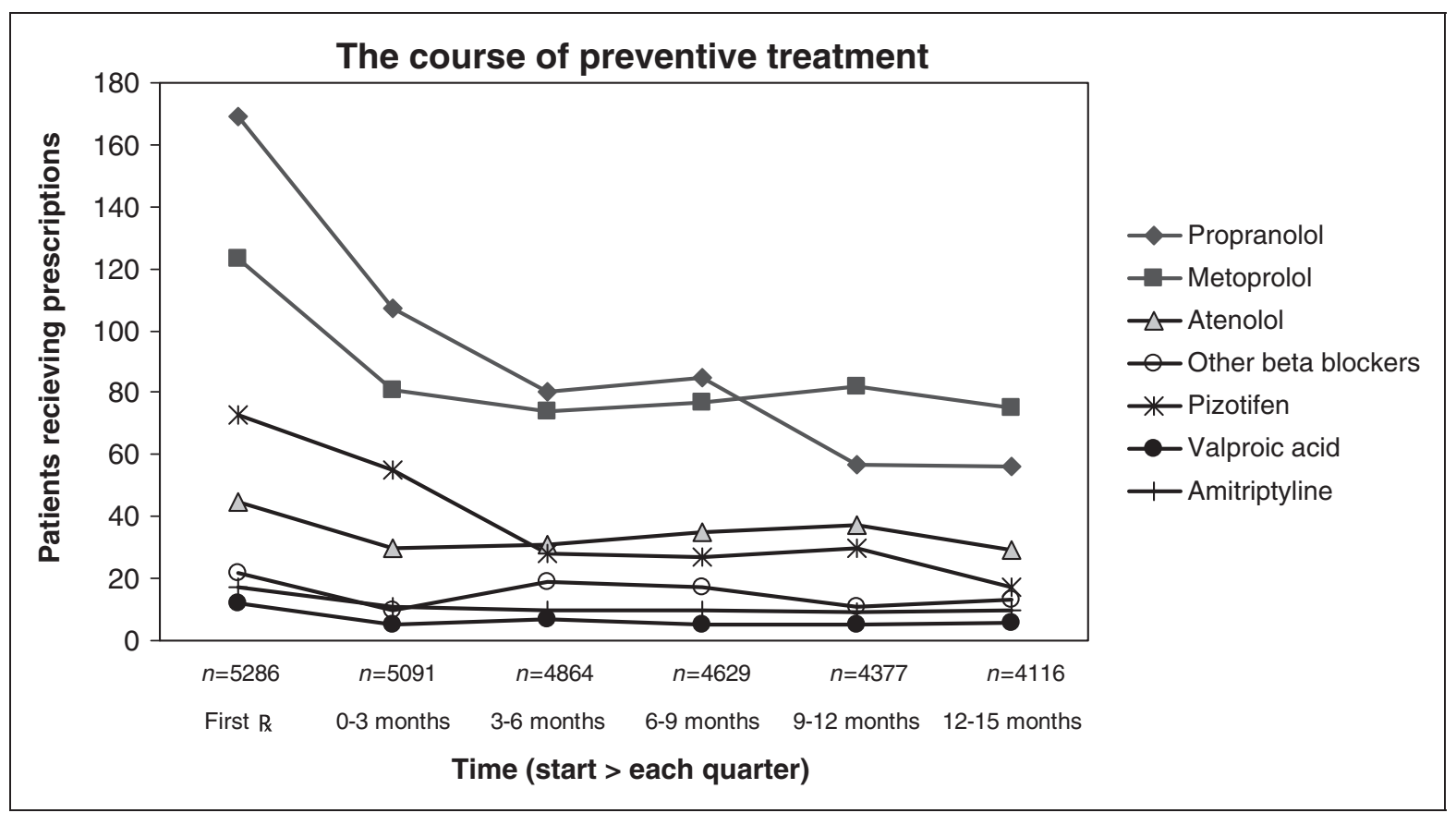

Figure 3. The course of preventive treatment.

Each line represents a preventive treatment. On the $\mathrm{X}$-axis the total number of patient in follow-up is shown. $n$ : number.

In the recent study by Berger et al., based on a US health insurance claims database, continued use was lower. For example, for beta blocker use after six months, continued use was $43.1 \%$, compared to $59.6 \%$ in ours. However, there were various differences with our study. We used the volume of prescriptions, not only the simple amount of prescriptions. Our inclusion is based on a validated method for diagnosis; their inclusion was based also on medication use as such (leading to higher numbers of patients). Finally, their research period was shorter (two years) (28).

Preventive treatment is prescribed to only $4.8 \%$ of triptan users and to $4.2 \%$ of NSAID users (applies only to prescribed medication, not to OTCs). This finding differs from other studies in which triptan users have more preventive medication $(13,25)$. However, other studies are usually not based on clinical diagnosis.

In this study, using observational primary-care information, misclassification of migraine may have occurred. False-negative misclassification could have occurred if patients did not seek GP attention or if they were treated solely by a specialist. As neurologists in the Netherlands are treating $3 \%$ of migraine patients, the possibility of under-presenting of severe migraine patients will likewise only be small (13).

Of the 478,585 eligible people in the database, we identified 7525 certain and possible new migraine patients at an incidence rate of 4.05 per 1000 person years. These findings may not apply to the overall migraine population. In the EPR many characteristics of migraine, such as the presence of aura and relation to the menstrual period, is often insufficiently described. This most likely explains the low proportion of patients with aura $(20.5 \%)$ and the probable under-reporting of MRM migraine (9.1\%) and typical AWoH (3.0\%).

As preventive treatment for $\mathrm{AWoH}$ is not applicable or controversial, we did not include this diagnosis in our study. However, we included $\mathrm{MOH}$, since we had sufficient observation time for each subject to reliably assess the temporal relationship between medication overuse and diagnosis of migraine (usually migraine, rarely tension-type headache, as recorded in the EPR). Including patients with $\mathrm{MOH}$ in the analysis may have caused some contamination by tension-type headache but, given the numbers, it will not have a major impact on the estimates.

This study shows a long-lasting and continued use for nine to 12 months of preventive medication in $56 \%$ of patients starting on preventive medication for migraine. Although this real-life study cannot be compared with studies in an experimental setting, in our study the frequency of treatment withdrawal is much higher, especially for beta blockers (31). In usual care treatment withdrawal can be interpreted, at least for a part, as non-response. The response rate in experimental trials is around $50 \%$, which is in line with the withdrawal rate in our study (31). The guideline compliance in our study was much better than in an older study (1995-1998) (25). 
The absence of differences in treatment patterns between types of migraine and between population subgroups suggests that this study gives no indication of extra attention in the area of preventive treatment for any specific group of patients.

Despite many efforts to improve the treatment of migraine and an otherwise well-used headache guideline in the Netherlands that promotes preventive treatment, there is much room for improvement. The compliance with treatment guidelines has improved, but the actual number of patients on preventive treatment remains low.

Qualitative research could give more insight into the reasons behind the limited use of preventive medication in younger migraine patients, the extended duration between diagnosis and start of preventive treatment, and why and how prescribed attack treatment interferes with preventive treatment (e.g. whether patient and physicians accept only one treatment for one illness at the same time).

This may point the way toward better explanation and defense of preventive treatment to expand its use, as well as encouraging more than one attempt using alternative medication.

Although compliance with preventive treatment has improved and many patients have experience with it, preventive treatment still needs attention in primary care. Further research into the underlying reasons and motives of patients and physicians is desirable. Firstly, the greatest gain would be made if more patients would consult their GPs when they have migraine attacks, thereby leading to more migraine diagnoses. Secondly, more and/or repeated diagnoses followed by actively inviting migraine patients for a personal consultation to discuss preventive treatment would lead to even more improvement.

\section{Clinical implications}

- A limited number of patients are current users of preventive treatment (13\%), but many patients have prior use $(44 \%)$.

- After diagnosis there is often an extended time before preventive treatment is applied, on average more than four years.

- Often there is only one attempt at preventive therapy.

- The continuation in time of preventive treatment equals study level.

\section{Funding}

This research received no specific grant from any funding agency in the public, commercial, or not-for-profit sectors.

\section{Conflicts of interest}

Drs Dekker, Dieleman, Knuistingh Neven and Assendelft have nothing to declare. Dr Ferrari has received in the past five years, grants and consultancy or industry support from Colucid, GlaxoSmithKline, Linde, MAP, Medtronic, Menarini, and Merck, and independent support from the Netherlands Organisation for Scientific Research (NOW), National Institutes of Health (NIH), European Community FP6, Biomed EC, the Dutch Heart and Brain Foundations, and Leiden University Medical Center (LUMC).

\section{References}

1. Ferrari MD. Migraine. Lancet 1998; 351: 1043-1051.

2. World Health Organization. Atlas of headache disorders and resources in the world 2011. Geneva: WHO, 2011.

3. Grol MH, Neven AK, Pijnenborg L, et al. Summary of the practice guideline 'Headache' from the Dutch College of General Practitioners [in Dutch]. Ned Tijdschr Geneeskd 2006; 150: 305-309.

4. Couturier EG, Bomhof MA, Gooskens R, et al. Richtlijnen diagnostiek en behandeling chronisch recidiverende hoofdpijn zonder neurologische afwijkingen. Utrecht: Nederlandse Vereniging voor Neurologie, 2008.

5. Evers S, Afra J, Frese A, et al. EFNS guideline on the drug treatment of migraine-report of an EFNS task force. Eur J Neurol 2006; 13: 560-572.

6. Modi S and Lowder DM. Medications for migraine prophylaxis. Am Fam Physician 2006; 73: 72-78.

7. Lipton RB and Bigal ME. Ten lessons on the epidemiology of migraine. Headache 2007; 47(Suppl 1): S2-S9.

8. Lipton RB, Bigal ME, Diamond M, et al. Migraine prevalence, disease burden, and the need for preventive therapy. Neurology 2007; 68: 343-349.

9. Lipton RB, Scher AI, Kolodner K, et al. Migraine in the United States: Epidemiology and patterns of health care use. Neurology 2002; 58: 885-894.

10. Diamond S, Bigal ME, Silberstein S, et al. Patterns of diagnosis and acute and preventive treatment for migraine in the United States: Results from the American Migraine Prevalence and Prevention study. Headache 2007; 47: 355-363.

11. Lucas C, Chaffaut C, Artaz MA, et al. FRAMIG 2000: Medical and therapeutic management of migraine in France. Cephalalgia 2005; 25: 267-279.

12. Kol CM, Dekker F, Neven AK, et al. Acceptance or rejection of prophylactic medicine in patients with migraine: A cross-sectional study. Br J Gen Pract 2008; 58: $98-101$. 
13. Dekker F, Wiendels N, de Valk V, et al. Triptan overuse in the Dutch general population: A nationwide pharmaco-epidemiology database analysis in 6.7 million people. Cephalalgia 2011; 31: 943-952.

14. Wiendels NJ, Knuistingh Neven A, Rosendaal FR, et al. Chronic frequent headache in the general population: Prevalence and associated factors. Cephalalgia 2006; 26 : 1434-1442.

15. Wiendels NJ, van Haestregt A, Knuistingh Neven A, et al. Chronic frequent headache in the general population: Comorbidity and quality of life. Cephalalgia 2006; 26: 1443-1450.

16. Okkes I, Jamoulle M, Lamberts H, et al. ICPC-2-E: The electronic version of ICPC-2. Differences from the printed version and the consequences. Fam Pract 2000; 17: 101-107.

17. International Classification Committee of WONCA. ICPC-2 International classification of primary care, 2nd edn. Oxford: Oxford University Press, 1998.

18. World Health Organization Collaborating Centre for Drug Statistics Methodology. Guidelines for ATC classification and DDD assignment. Oslo: WHO, 1998.

19. Vlug AE, van der Lei J, Mosseveld BM, et al. Postmarketing surveillance based on electronic patient records: The IPCI project. Methods Inf Med 1999; 38: 339-344.

20. Headache Classification Subcommittee of the International Headache Society. The International Classification of Headache Disorders. Cephalalgia 2004; 24(Suppl 1): 9-160.

21. Dekker F, Knuistingh NA, Andriesse B, et al. Prophylactic treatment of migraine; the patient's view, a qualitative study. BMC Fam Pract 2012; 13: 13.

22. Schuurmans A and van Weel C. Pharmacologic treatment of migraine. Comparison of guidelines. Can Fam Physician 2005; 51: 838-843.
23. Antonaci F, Dumitrache C, De Cillis I, et al. A review of current European treatment guidelines for migraine. J Headache Pain 2010; 11: 13-19.

24. Rahimtoola H, Buurma $H$, Tijssen $C C$, et al. Incidence and determinants of migraine prophylactic medication in the Netherlands. Eur J Clin Pharmacol 2002; 58: 149-155.

25. Rahimtoola H, Buurma H, Tijssen CC, et al. Migraine prophylactic medication usage patterns in the Netherlands. Cephalalgia 2003; 23: 293-301.

26. Cevoli S, D'Amico D, Martelletti P, et al. Underdiagnosis and undertreatment of migraine in Italy: A survey of patients attending for the first time 10 headache centres. Cephalalgia 2009; 29: 1285-1293.

27. Lafata J, Tunceli O, Cerghet M, et al. The use of migraine preventive medications among patients with and without migraine headaches. Cephalalgia 2010; 30: 97-104.

28. Berger A, Bloudek LM, Varon SF, et al. Adherence with migraine prophylaxis in clinical practice. Pain Pract 2012; 12: 541-549.

29. Valade D, Lantéri-Minet M, Radat F, et al. Clinical determinants of migraine preventive treatment: Contribution of SMILE, an observational survey of primary care migraine management in France. Cephalalgia 2010; 30: 1207-1213.

30. Zielman R, Veenstra P, Zwet E, et al. How general practitioners treat migraine patients: Evaluation of a headache guideline. Cephalalgia 2012; 32: 908-915.

31. Linde $\mathrm{K}$ and Rossnagel K. Propranolol for migraine prophylaxis. Cochrane Database Syst Rev 2004: CD003225. 